\title{
PERFIL DOS PACIENTES ONCOLÓGICOS ATENDIDOS EM UMA UNIDADE DE EMERGÊNCIA
}

\author{
PROFILE OF THE ONCOLOGIC PATIENTS TREATED AT \\ THE EMERGENCY UNIT
}

\section{PERFIL DE LOS PACIENTES ONCOLÓGICOS TRATADOS EN UNA UNIDAD DE EMERGENCIA}

\author{
Ana Paula Boaventura* \\ Cleuza Aparecida Vedovato ${ }^{* *}$ \\ Francisleine FrancK dos SANTOS ${ }^{* * *}$
}

\begin{abstract}
RESUMO
Objetivo: Analisar o perfil dos pacientes oncológicos atendidos na Unidade de Emergência de um hospital público estadual no interior do Estado de São Paulo - Brasil. Material e método: Este é um estudo exploratório, retrospectivo. Foram utilizados os prontuários dos pacientes atendidos no departamento de emergência no ano de 2012. Resultados: Foram atendidos 172 pacientes oncológicos em um ano, 62,2\% (107) do sexo masculino com média de idade de 62 anos, sendo 69,8\% (120) pacientes no estágio inicial ou intermediário da doença, 97,6\% (168) dos pacientes fizeram quimioterapia e 39,5\% (68) radioterapia e $86 \%$ (148) destes pacientes oncológicos não apresentaram metástase, sendo 57,5\% (99) tiveram alta do serviço de emergência. Quanto aos sinais e sintomas que levaram estes pacientes procurarem o serviço de emergência estão a dor 83,1\% (143), náuseas e vômitos para 67,4\% (116), febre 26,1\% (45) e fraqueza apresentada por 11,6\% (20) pacientes. Os tumores eram de origem gastrointestinal para 27,3\% (47), pulmão 18,6\% (32) e mama 16,9\% (29), sendo utilizados analgési$\cos 94,7 \%$ (163) e antieméticos 79,6\% (137), por via endovenosa periférica 45\% (77). Conclusão: Concluiu-se que é pequena a prevalência de pacientes oncológicos nesta unidade de emergência, e que o tratamento dos sintomas em pacientes com câncer é uma preocupação multiprofissional complexa e os serviços de emergência devem ter estrutura física e recursos humanos qualificados para atender os pacientes.
\end{abstract}

Palavras chave: Enfermagem em emergência, serviços médicos de emergência, oncologia.

\begin{abstract}
Objective: To analyze the profile of oncology patients treated at the Emergency Unit of a public hospital in the State of São Paulo - Brazil. Method: This is an exploratory, retrospective study. Medical records were used from patients seen in the emergency unit in the year 2012. Results: 172 cancer patients were treated at one year, $62.2 \%$ (107) were male with a mean age of 62 years, $69.8 \%$ (120) patients in the initial or intermediate stage of the disease, $97.6 \%$ (168) of the patients received chemotherapy and 39.5\% (68) radiation and $86 \%$ (148) of these

\footnotetext{
*Enfermeira, Professor Doutor da Faculdade de Enfermagem da Universidade Estadual de Campinas, Brasil. E-mail: apboa@ unicamp.br

${ }^{*}$ Enfermeira, Mestre. Professor da Faculdade de Enfermagem da Universidade Estadual de Campinas, Brasil. E-mail: vedovato@unicamp.br

${ }^{* * *}$ Enfermeira do Serviço de Emergências Hospital Estadual Sumaré, Brasil. E-mail: franckleyne@yahoo.com.br
} 
patients did not have cancer metastasis and 57.5\% (99) were discharged from the emergency unit. The signs and symptoms that led these patients to seek emergency services are pain $83.1 \%$ (143), nausea and vomiting to $67.4 \%$ (116), fever $26.1 \%$ (45) and weakness presented by $11.6 \%$ (20) cases. Tumors were gastrointestinal disturbances to $27.3 \%$ (47), lungs $18.6 \%$ (32) and breast $16.9 \%$ (29), analgesics being used $94.7 \%$ (163) and antiemetics 79,6\% (137), peripheral intravenous $45 \%$ (77). Conclusion: It was concluded that there is little prevalence of cancer patients in this emergency unit, and the treatment of symptoms in cancer patients is a complex multidisciplinary concern and emergency services should have physical infrastructure and qualified human resources to serve patients.

Key words: Emergency nursing, emergency medical services, oncology.

\section{RESUMEN}

Objetivo: Analizar el perfil de los pacientes oncológicos atendidos en el Servicio de Urgencias de un hospital público en el estado de São Paulo - Brasil. Material y método: Estudio exploratorio, retrospectivo. Fueron utilizados los registros médicos de los pacientes atendidos en el servicio de urgencias en el año 2012. Resultados: Fueron tratados 172 pacientes con cáncer en un año; el 62,2\% (107) eran hombres con una edad media de 62 años, encontrándose el 69,8\% (120) de los pacientes en etapa inicial o intermedia de la enfermedad; el 97,6\% (168) de los pacientes recibieron quimioterapia, 39,5\% (68) radioterapia y el $86 \%$ (148) de los pacientes no tenían metástasis del cáncer, siendo 57,5\% (99) dados de alta del servicio de urgencias. Los signos y síntomas que llevaron a estos pacientes a buscar los servicios de emergencia fueron el dolor 83,1\% (143), náuseas y vómitos $67,4 \%$ (116), fiebre $26,1 \%$ (45) y debilidad $11,6 \%$ (20). Los tumores fueron de origen gastrointestinal $27,3 \%$ (47), pulmón 18,6\% (32), mama 16,9\% (29), siendo los analgésicos utilizados en 94,7\% (163) y antieméticos $79,6 \%$ (137) por via venosa periférica 45\% (77). Conclusión: Hay poca prevalencia de pacientes con cáncer en este servicio de urgencias, el tratamiento de los síntomas en pacientes con cáncer es una preocupación de servicios multidisciplinarios compleja y los servicios de emergencia deben tener la infraestructura y los recursos humanos cualificados para atender a los pacientes.

Palabras clave: Enfermería de urgencia, servicios médicos de urgencia, oncología.

Fecha recepción: 17/03/14 Fecha aceptación: 07/07/15

\section{INTRODUÇÃO}

As estimativas mundiais para 2030, segundo a Organização Mundial da Saúde (OMS) aponta 21,4 milhões de casos novos de câncer e 13,2 milhões de mortes por câncer, em consequência do crescimento e do envelhecimento da população, em contrapartida a redução na mortalidade infantil e nas mortes por doenças infecciosas $(1,2)$.

O câncer é a segunda principal causa de morte no mundo, depois da doença cardiovascular. Com o aumento do tamanho da população idosa, o número de novos casos de câncer e os efeitos secundários relacionados com o tratamento vem aumentando progressivamente $(1,2)$.

A maioria dos pacientes com câncer experimentam pelo menos uma emergência durante a sua doença e o desenvolvimento do novos tratamentos e estratégias para pacientes oncológicos, tem resultado em uma vida prolongada e um aumento do número de emergência que possam ser vivenciadas em decorrência destes novos tratamentos (3).

Algumas emergências oncológicas tem início súbito ou podem demorar meses a desenvolver e manifestam-se abruptamente, ao passo que outras se manifestam em algumas 
horas, resultando em complicações e agravamentos da doença, podendo levar a morte (3, 4).

São nos serviços de urgência e emergências que aparecem os pacientes com as patologias de maior incidência na população, sendo elas emergências clínicas ou cirúrgicas, envolvendo os politraumatismos, as doenças cardiovasculares e cerebrovasculares e as doenças oncológicas que hoje são a segunda maior causa de morte (superadas apenas pelas doenças do aparelho circulatório e pelas causas externas / violência). Estima-se que em bem pouco tempo o câncer já seja a principal causa de morte no mundo se medidas preventivas não forem instaladas efetivamente na população $(3,4)$.

Os motivos que levam ao crescimento da incidência do câncer são o aumento da expectativa de vida da população em geral, associada a maior exposição a fatores de risco $(1,2)$.

A oncologia é a especialidade médica que estuda as neoplasias malignas, e a forma como estas patologias se desenvolvem no organismo, buscando seu tratamento. A oncologia é dividida em clínica e cirúrgica, sendo que a clínica atua principalmente através de quimioterapia e radioterapia e a cirúrgica atua na retirada de tecido tumoral do organismo por meio de ato cirúrgico, podendo ser: curativa, paliativa ou adjuvante $(1,2)$.

A maioria das emergências oncológicas podem ser categorizados como metabólica (hipercalcemia, síndrome de inadequado hormona anti-diurética, síndroma de lise tumoral), hematológica (neutropenia febril), estrutural (medula espinhal, compressão de nervos periféricos, derrame pericárdico maligno) ou efeitos colaterais de agentes quimioterápicos (diarréia, extravasamento) (3, 4).

O diagnóstico precoce e tratamento adequado de pacientes com câncer no serviço de emergência são eficazes na restauração da qualidade de vida. Portanto, serviços de emergência desempenham um papel impor- tante no atendimento para este grupo de pacientes $(3,4)$.

No gerenciamento da doença oncológica, a fase de agravamento e cuidado crítico caracteriza-se pela instabilidade emocional e clínica do cliente, com exacerbação de sintomas que pioram a qualidade de vida, podendo representar o complexo processo de morrer e da morte. Nessa fase, em muitas situações, o cliente precisa ser hospitalizado, e o hospital pode acabar sendo o local do óbito (5).

Os cuidados direcionados ao fim da vida acontecem, em sua maioria, no hospital, em detrimento do limitado número de equipes de saúde capacitadas para o atendimento desses pacientes oncológicos, no âmbito da atenção básica. Apesar desse nível de assistência contar com programas específicos de atendimento domiciliar, seguindo modelo de gestão do Sistema Único de Saúde (SUS), dados de 2010, divulgados pelo DATASUS, mostram que o hospital é o principal local para a ocorrência do óbito, chegando a $80 \%$ dos casos nas mortes por câncer $(1,2,5)$.

Pretende-se com este estudo, identificar o perfil dos pacientes oncológicos atendidos em uma unidade de emergência de um hospital público, visando contribuir e chamar atenção dos profissionais de saúde para a especificidade do atendimento a estes pacientes nos serviços de emergência.

\section{MATERIAL E MÉTODO}

Trata-se de um estudo quantitativo com delineamento retrospectivo de carácter exploratório. Realizado em uma unidade de emergência, no interior do Estado de São Paulo. Trata-se de um hospital público no modelo de gestão e organização do serviço de saúde (OSS) do governo do Estado de São Paulo.

Esta unidade de emergência recebe em média 600 pacientes adultos por mês, em especialidades clínicas ecirúrgicas, tendo em 
seu quadro de funcionários sete enfermeiros, dois médicos clínicos por plantão, um médico cirurgião (residente cirurgia) por dia, 42 técnicos de enfermagem, dois fisioterapeutas, um auxiliar administrativo, um nutricionista, um assistente social e oito recepcionistas.

Foram utilizados os dados dos atendimentos realizados aos pacientes com patologias oncológicas na unidade de emergência referenciada no ano de 2012. Estes dados são armazenados em banco de dados da instituição e prontuário eletrônico onde foram utilizados única e exclusivamente para realização deste trabalho.

Critérios de inclusão: fizeram parte deste estudo todos os dados do banco de dados referentes a atendimentos dos pacientes com diagnósticos de patologias oncológicas que procuraram a unidade de primeiro de janeiro a 31 de dezembro de 2012.

Critérios de exclusão: foram excluídos dados incompletos e imprecisos e de pacientes sem diagnósticos de patologias oncológicas.

O projeto de pesquisa foi submetido ao Comitê de Ética em Pesquisa do Hospital, do Comitê de ética em Pesquisa da Faculdade de Ciências Médicas da Universidade Estadual de Campinas (FCM/Unicamp), filiado ao Comitê Nacional de Ética em Pesquisa - CONEP e aprovado sob o parecer $293.407 \mathrm{em}$ 28/05/2013.

A coleta dos dados foi realizada do sistema de prontuário eletrônico da instituição e da unidade de emergência, este sistema fornece os dados de todos os atendimentos realizados. Para este estudo foram selecionados os dados dos pacientes com diagnósticos oncológicos atendidos no ano de 2012.

As variáveis coletadas para analisar o perfil destes pacientes foram: idade, sexo, estágio da doença oncológica, número de cirurgias realizadas devido ao diagnóstico oncológico, presença de comorbidades, tempo do diagnóstico oncológico, se fez tratamento quimioterápico ou radioterápico, se há presença de metástase, quais os sinais e sintomas que levaram ao atendimento de emergência, quais as condutas realizadas, quais medicações utilizadas, vias de administração e qual foi o desfecho dos atendimentos na unidade de emergência.

\section{RESULTADOS}

Foram atendidos um total de 10.792 pacientes adultos nesta unidade de emergência no ano de 2012 e, destes, 172 pacientes apresentavam patologias oncológicas. Na Tabela 1 estão apresentadas as características clínicas e demográficas identificadas nos pacientes oncológicos adultos.

Identificou-se uma média de idade de 62 anos, sendo 62,2\% (107) do sexo masculino, apresentavam além do câncer outras comorbidades 75,6\% (130), tendo realizado pelo menos uma cirurgia devido ao câncer 77,9\% (134) e 28,4\% (49) pacientes evoluíram a óbito em um período de 24 horas após o atendimento na unidade de emergência.

Os sinais e sintomas que estes pacientes apresentavam estão descritos na Tabela 2, verificou-se que alguns pacientes apresentaram mais de um sinal e sintoma no mesmo atendimento, sendo dor 83,1\% (143), náuseas e vômitos $67,4 \%$ (116) e febre $26,1 \%(45)$ predominantemente apresentados como queixa principal na admissão no serviço de emergência.

Outros sinais e sintomas $9,8 \%$ (17) foram sangramentos, lesões de pele e mucosas e dor abdominal.

Quanto a localização primária do tumor nestes pacientes os tumores gastrointestinais e pulmonares foram predominantes, seguidos pelo câncer de mama, como podemos observar na Tabela 3 a seguir.

Diante dos sinais e sintomas são necessárias intervenções para resolver ou alivia-los, na Tabela 4 a seguir estão descritos os principais agentes farmacológicos utilizados nestes pacientes durante os atendimentos na unidade de emergência. 
Tabela 1. Características demográficas e clínicas dos pacientes oncológicos atendidos na unidade de emergência (Campinas, 2013).

\begin{tabular}{|c|c|c|}
\hline Características & $\mathrm{n}=172$ & $\%$ \\
\hline Idade (anos, média $\pm \mathrm{DP}$ ) & $62 \pm 15,4$ & \\
\hline \multicolumn{3}{|l|}{ Sexo } \\
\hline Masculino & 107 & 62,2 \\
\hline Feminino & 65 & 37,7 \\
\hline \multicolumn{3}{|l|}{ Estágio da doença } \\
\hline Inicial/intermediária & 120 & 69,8 \\
\hline Terminal & 52 & 30,2 \\
\hline \multicolumn{3}{|l|}{ Número de cirúrgias } \\
\hline Até uma & 134 & 77,9 \\
\hline Acima de duas & 38 & 22,1 \\
\hline \multicolumn{3}{|l|}{ Comorbidades } \\
\hline Sim & 130 & 76 \\
\hline Não & 42 & 24 \\
\hline \multicolumn{3}{|l|}{ Desfecho dos atendimentos } \\
\hline Internação & 24 & 14,1 \\
\hline Alta & 99 & 57,5 \\
\hline Óbito & 49 & 28,4 \\
\hline \multicolumn{3}{|l|}{ Tempo do diagnóstico } \\
\hline 0 a 12 meses & 87 & 50,6 \\
\hline 13 a 24 meses & 26 & 15,1 \\
\hline 25 a 36 meses & 16 & 9,3 \\
\hline Mais de 36 meses & 43 & 25,0 \\
\hline \multicolumn{3}{|l|}{ Quimioterapia } \\
\hline Sim & 168 & 97,6 \\
\hline Não & 4 & 2,4 \\
\hline \multicolumn{3}{|l|}{ Radioterapia } \\
\hline Sim & 68 & 39,5 \\
\hline Não & 104 & 60,5 \\
\hline \multicolumn{3}{|l|}{ Metástase } \\
\hline Sim & 24 & 14 \\
\hline Não & 148 & 86 \\
\hline
\end{tabular}

Tabela 2. Sinais e sintomas apresentados pelos pacientes oncológicos atendidos na unidade de emergência (Campinas, 2013).

\begin{tabular}{lcc}
\hline Sinais e sintomas & $\mathbf{N}$ & $\%$ \\
\hline Dor & 143 & 83,1 \\
Naúsea/vômitos & 116 & 67,4 \\
Febre & 45 & 26,1 \\
Fraqueza & 20 & 11,6 \\
Dispnéia & 14 & 8,1 \\
Inapetência & 12 & 6,9 \\
Derrame pleural & 10 & 5,8 \\
Infecção & 9 & 5,2 \\
Outros & 17 & 9,8 \\
\hline
\end{tabular}


Tabela 3. Localização primária do tumor (Campinas, 2013).

\begin{tabular}{lcc}
\hline Localização primária do tumor & $\mathbf{n = 1 7 2}$ & $\mathbf{\%}$ \\
\hline Gastrointestinal & 47 & 27,3 \\
Pulmão & 32 & 18,6 \\
Mama & 29 & 16,9 \\
Próstata & 26 & 15,1 \\
Ovário & 16 & 9,3 \\
Linfoma & 10 & 5,8 \\
Cabeça e pescoço & 5 & 2,9 \\
Pele & 4 & 2,3 \\
Cérebro & 3 & 1,7 \\
\hline
\end{tabular}

Tabela 4. Terapia medicamentosa e vias de administração utilizados para os pacientes oncológicos na unidade de emergência (Campinas, 2013).

\begin{tabular}{lcc}
\hline Fármacos & $\mathbf{n = 1 7 2}$ & $\mathbf{\%}$ \\
\hline Analgésicos & 163 & 94,7 \\
Antieméticos & 137 & 79,6 \\
Antiagregantes plaquetários & 89 & 51,7 \\
Profiláticos úlcera gástrica & 77 & 44,8 \\
Antibioticoterapia & 64 & 37,2 \\
Sedativos hipnóticos & 48 & 27,9 \\
Opióides & 27 & 15,7 \\
Antipsicóticos & 14 & 8,1 \\
Anti-histamínicos & 14 & 8,1 \\
Neurolépticos & 11 & 6,4 \\
Benzodiazepínicos & 9 & 5,2 \\
\hline Vias de administração & $\mathbf{n}=\mathbf{1 7 2}$ & $\mathbf{\%}$ \\
\hline Acesso venoso periférico & 77 & 44,7 \\
Acesso venosos central & 31 & 18,0 \\
Hipodermóclise & 43 & 25,0 \\
Outras & 21 & 12,2 \\
\hline
\end{tabular}

Alguns outros procedimentos realizados foram: nutrição enteral, sonda nasogástrica aberta, intubação orotraqueal em ventilação mecânica, traqueostomia, endoscopia, sondagem vesical de demora, paracentese e transfusão sanguínea.

Com relação às medicações prescritas: 94,7\% (163) pacientes possuíam analgesia prescrita (dipirona ou tramadol); 79,6\%
(137) antieméticos (metoclopramida ou ondansentrona); 51,7\% (89) com profilaxia TEP (tromboembolismo pulmonar) prescrita (enoxaparina ou AAS); 44,8\% (64) profilaxia úlcera gástrica (ranitidina ou omeprazol); 27,9\% (48) com antibioticoterapia; 15,7\% (27) com opióides (morfina); 8,1\% (14) com antipsicóticos e anti-histamínicos prescritos (clorpromazina e prometazina); 8,1\% (14) 
com neurolépticos prescrito (haldol); 6,4\% (11) com benzodiazepínico e 5,2\% (9) com sedação (fentanil + midazolan) em bomba de infusão contínua.

O perfil dos pacientes oncológicos atendidos nesta unidade de emergência é de $62,2 \%$ (107) do sexo masculino com média de idade de 62 anos, sendo 69,8\% (120) pacientes no estágio inicial ou intermediário da doença, diagnosticados em até 12 meses 50,6\% (87), onde $97,6 \%$ (168) dos pacientes fizeram quimioterapia e $39,5 \%$ (68) radioterapia e $86 \%$ (148) destes pacientes oncológicos não apresentaram metástase, sendo $57,5 \%$ (99) tiveram alta do serviço de emergência.

Quanto aos sinais e sintomas que levaram estes pacientes procurarem o serviço de emergência estão a dor $83,1 \%$ (143), náuseas e vômitos para $67,4 \%$ (116), febre $26,1 \%$ (45) e fraqueza apresentada por $11,6 \%$ (20) pacientes. Os tumores eram de origem gastrointestinal para $27,3 \%$ (47), pulmão $18,6 \%$ (32) e mama $16,9 \%$ (29), sendo utilizados mais analgésicos 94,7\% (163) e antieméti$\cos 79,6 \%$ (137), como via de administração mais utilizada o acesso venoso periférico $45 \%$ (77) nestes pacientes oncológicos.

\section{DISCUSSÃO E CONCLUSÃO}

Segundo as estimativas da OMS, até 2030, as pessoas com 65 anos ou mais representarão $70 \%$ do total de indivíduos diagnosticados com câncer, o que caracteriza um universo populacional importante onde medidas preventivas são fundamentais para evitar maior sofrimento e piora da qualidade de vida da população $(1,2)$.

Os serviços de emergência recebem diariamente pacientes com complicações de doenças de maior incidência na população e no caso de pacientes oncológicos alguns fatores relacionados a doença e ao tratamento como anorexia/caquexia, desnutrição, anemia, alterações metabólicas, mobilidade física re- duzida, distúrbios circulatórios e até mesmo alterações neurológicas, que acarretam a falência da pele e demais órgãos estão presentes em pacientes com câncer, assim como o comprometimento da resposta imunológica, especialmente naqueles em tratamento com corticoides e imunossupressores $(3,6)$.

$\mathrm{O}$ número de pessoas em tratamento oncológico que estão sendo atendidas nos serviços de emergência aumenta progressivamente e diariamente, e os enfermeiros são os profissionais que oferecem o primeiro atendimento para a maioria dos pacientes e precisam ser capazes de reconhecer as emergências oncológicas para iniciar urgente avaliação e encaminhamento para a equipe de emergência oncológica a fim de que o cuidado específico possa ser imediatamente instituído (3).

Em estudo retrospectivo semelhante também realizado em uma unidade de emergência com 408 pacientes com câncer que foram atendidos em um serviço de emergência entre janeiro e dezembro de 2011, identificou um perfil de pacientes atendidos de 240 $(58,8 \%)$ do sexo masculino e $168(41,2 \%)$ do sexo feminino, com media de idade de 57,9 anos (variação 19-87). Em relação estadiamento do câncer, 266 pacientes $(65,3 \%)$ tiveram metástase e $142(34,7 \%)$ tinham doença local e loco-regional. A taxa de internação foi de $59,6 \%$. Os sintomas mais comuns foram a falta de ar $(23,2 \%)$, dor $(17,8 \%)$, febre (14,2\%), e náusea / vômito $(14,4 \%)$. Os locais mais comuns de câncer foram os de pulmão (32,5\%), sistema gastrointestinal $(25,4 \%)$, e mama (9,3\%). Destes, 191 (46,8\%) pacientes morreram após a admissão na unidade de emergência, e a sobrevida destes pacientes em 1 ano foi de 7,3 meses (4).

Em revisão integrativa da literatura que objetivou caracterizar artigos abordando a qualidade de vida relacionada à saúde (QVRS) de pacientes com câncer avançado nas literaturas nacional e internacional e sumarizar os fatores contribuintes para a melhora ou a piora, identificou em 13 trabalhos 
sinais e sintomas preditores de baixa qualidade de vida, como dor, fadiga, distúrbio do sono, depressão, alterações nutricionais, entre outros. Os resultados mostram que manifestações clínicas inerentes ao câncer são fatores que podem rebaixar a qualidade de vida do paciente, enquanto os benefícios físicos, psicológicos e espirituais resultantes de intervenções terapêuticas, podem promover significativa melhora (7).

Em estudo realizado no Hospital Universitário Regional do Norte do Paraná (HURNP) com pacientes em cuidados paliativos, visando avaliar a situação de dor e terapêutica empregada do doente em fase terminal, identificou como resultado em 39 prontuários que para analgesia 95\% tinham apenas Dipirona endovenosa prescrita, dos quais $78,4 \%$ estavam prescritos no esquema "se necessário" e o restante $(21,6 \%)$ prescritos com horário fixo. Apenas dois pacientes faziam uso de opióide fraco, em horários regulares e dois pacientes usavam opióides fortes em horário fixo. Outras drogas foram encontradas que podem ter sido utilizados ou não como adjuvantes para o alívio da dor, como analgésico comum, antiinflamatórios esteróides e anticonvulsivantes. Não havia registro de dor referida pelos doentes, uma vez que eles se encontravam impossibilitados de comunicar-se verbalmente e a instituição não utiliza escalas para avaliação sistemática da dor (8).

Nos pacientes oncológicos, a dor é fator desencadeante de desconforto e aparece como queixa principal nos serviços de emergência, associada a náuseas e vômitos e febre, e levam consequentemente à utilização de analgésicos, antitérmicos e antieméticos administrados por via endovenosa.

Em estudo com o objetivo de identificar como está a assistência e o conhecimento da equipe de enfermagem frente à dor oncológica para aprofundar o conhecimento sobre as práticas de cuidados de enfermagem ao paciente oncológico com dor, identificouse que a dor é um dos principais males que acometem o paciente com câncer, no Brasil a estimativa é que 62 a 90\% dos pacientes com câncer apresentam algum tipo de dor, e realizam inadequadamente o controle e o tratamento, sendo que 24,5 a 46,6\% têm sua dor inadequadamente controlada (9).

É necessário prática para atender um paciente com dor oncológica, pois deve haver uma ampla abordagem e a sua avaliação é o ponto fundamental para o planejamento do cuidado, exigindo do profissional de saúde suporte educacional para o manuseio e gerenciamento dessa sensação álgica (9).

Neste estudo 83,13\% (143) pacientes oncológicos apresentavam dor como queixa principal, que os levaram ao serviço de emergência. Assim como as náuseas e vômitos podem ser induzidos pelo tratamento quimioterápico e estes pacientes podem procurar os serviços de emergência devido a estes sintomas.

Estudos sugerem que a profilaxia com antieméticos devem ser instituídas por meio de protocolos e diretrizes, em pacientes que fazem tratamento quimioterápico a fim de diminuir a ocorrência de náuseas e vômitos nestes pacientes. Em estudo realizado avaliando os prontuários de 100 pacientes oncológicos identificou que 94\% receberam profilaxia antiemética do primeiro ao quarto dia de tratamento com redução expressiva dos sintomas (10).

Em estudo realizado com a finalidade de identificar os diagnósticos de enfermagem para pacientes hospitalizados em cuidados paliativos e quais as principais intervenções de enfermagem são realizadas para estes pacientes, através de estudo retrospectivo e quantitativo realizado em um hospital público do Rio de Janeiro - Brasil, com 280 registros analisados no $2^{\circ}$ Semestre de 2010, identificou-se dentre as intervenções de enfermagem a administração de medicações por via intramuscular ou subcutânea prescrita para apenas 27 (9,6\%) dos pacientes (5).

Os pacientes do Programa de Internação Domiciliar Interdisciplinar (PIDI) para pacientes oncológicos no Hospital Escola da 
Universidade Federal de Pelotas, no Município de Pelotas, Estado do Rio Grande do Sul, que apresentam intercorrências de maior gravidade são levados ao Pronto-Socorro local pelo Serviço de Atendimento Móvel de Urgência (SAMU - 192); quando retornam ao domicilio, permanecem sob os cuidados do PIDI, porém muitas vezes, os óbitos acontecem no domicílio e são acompanhados e constatados pela equipe do PIDI (11).

Dos 213 pacientes internados no programa no período de estudo, $56,0 \%$ eram do sexo masculino, $50,0 \%$ possuíam menos de 59 anos de idade e $41,0 \%$ se encontravam entre 60 e 70 anos. Quanto à cor da pele, 86,0\% eram brancos. A maioria dos pacientes era alfabetizada: $55,0 \%$ tinham estudo fundamental incompleto, $19,0 \%$ eram analfabetos e somente $1,0 \%$ apresentava ensino superior. Com relação à renda familiar, $44,0 \%$ recebiam até 1 salário mínimo e 40,0\%, 2 salários mínimos. Quanto à necessidade terapêutica no PIDI, a maioria dos pacientes apresentava finalidade paliativa $(95,0 \%)(11)$.

Quanto ao perfil dos pacientes oncológicos, em estudo realizado para avaliar duas modalidades de assistência a pacientes com câncer em cuidados paliativos, sendo uma modalidade de assistência domiciliar e outra modalidade de assistência ambulatorial, ambas com abordagem de equipe multiprofissional. Foram atendidos em um ano 1.451 pacientes em ambas modalidades, sendo em média 36 atendimentos por dia, $50,1 \%$ do sexo feminino e $49,9 \%$ masculino, com média de idade de 64 anos. Os principais sinais e sintomas atendidos foram náuseas e vômitos $40 \%$, constipação $35 \%$, dor em $79 \%$ dos pacientes e $94 \%$ com queixa de fraqueza. A origem inicial dos tumores foram pulmão $16 \%$, mama $13 \%$, hematológico $12 \%$ e gastrointestinal 12\% (12).

Os pacientes oncológicos precisam de infra-estrutura de atendimentos específicas para cuidar adequadamente deste paciente gravemente doente e morrendo, não sendo os serviços de emergências locais adequados para a realização desta assistência (12).

Ainda sobre pacientes oncológicos com agravamento dos sintomas, em estudo que avaliou em um ano o perfil e os custos dos atendimentos e das internações deste pacientes, identificou nos 120 pacientes, 62 homens e 58 mulheres, com média de idade de 61 anos, apresentaram um custo total médio de hospitalização por paciente foi de USD $12.962(1,244-138,877)$. E foram admitidos pela unidade de emergência com sintomas de dispneia $30 \%$, dor $24 \%$, náuseas e vômitos $11 \%$ e fadiga $14 \%$, necessitando de manobras de ressuscitação cardiopulmonar, unidade de terapia intensiva monitoramento e intubação. Os autores concluíram que a admissão de pacientes oncológicos em fase intermediária e terminal no serviço de emergência podem impactar o custo das internações destes pacientes oncológicos (13).

Outro problema que podem levar os pacientes oncológicos às unidades de emergência são as reações aos tratamentos e medicações instituídas, em estudo que objetivou avaliar os tipos, a causalidade e gravidade destas reações em pacientes oncológicos hospitalizados no período julho e dezembro de 2012, em 1.275 internações, identificou reações em 158 (12,4\%) pacientes com média de idade 61,3 anos, sendo $55,0 \%$ do sexo masculino e o tipo de câncer mais comum foi gastrointestinal $25,5 \%$, com $60 \%$ apresentando pelo menos uma comorbidade, sendo a hipertensão arterial sistêmica 39,5\% a mais comum. Foram identificadas as reações adversas as medicações, apresentando como principal sintoma a neutropenia febril, e combinações de medicamentos anti-hipertensivos com corticosteróides aumentado os riscos de potenciais interações medicamentosas entre estes pacientes com câncer (14).

Um estudo que avaliou o desfecho de atendimentos a pacientes em cuidados paliativos por um serviço domiciliar com equipes de profissionais especializados, identificou no período de 2009 a 2011, 3.109 pacientes, 
onde cerca de $80 \%$ tinham câncer e $78 \%$ receberam cuidados paliativos até o final da vida com atendimento domiciliar, $970(31,2 \%)$ foram atendidos no hospital e $896(28,9 \%)$ evoluíram a óbito no departamento de emergência. Os autores enfatizam a importância do atendimento domiciliar a estes pacientes visando a redução utilização de cuidados intensivos e óbitos hospitalares no final da vida (15).

Outro estudo que tinha como objetivo identificar e caracterizar as hospitalizações potencialmente evitáveis em pacientes com tumores gastrointestinais, entre dezembro de 2011 e julho de 2012, categorizaram os motivos das hospitalizações em "potencialmente evitáveis" ou "não evitáveis". Foram avaliadas 201 internações de pacientes com tumores gastrointestinais, idade média de 62 anos, e o câncer colo retal foi o diagnóstico mais comum (32\%). A maioria dos pacientes hospitalizados tinham metástase $(81 \%)$, sendo que $53 \%$ das internações foram atribuídas a sintomas de câncer, e $28 \%$ foram atribuídas a complicações do tratamento do câncer, os oncologistas médicos identificaram 39 (19\%) internações como potencialmente evitáveis. As hospitalizações foram categorizadas como potencialmente evitáveis para pacientes acima de 70 anos idade, já com propostas de cuidados paliativos e que estavam recebendo três ou mais esquemas de quimioterapia. Os sintomas mais comuns na admissão hospitalar foram febre/infecção (27\%), dor abdominal indiferenciada (12\%), e a obstrução do trato gastrointestinal (9\%) (16).

Tendo em vista a incidência progressiva mundial de casos de câncer nos últimos anos, ressalta-se a importância das ações preventivas e de controle, as quais são consideradas, atualmente, os mais importantes desafios científicos e de saúde pública, mas também o desenvolvimento e a infra-estrutura de serviços específicos para o atendimento aos pa- cientes oncológicos $(16,17)$.

Diante disso, é necessária a atuação de profissionais que proporcionem um atendimento efetivo e de boa qualidade tanto no âmbito hospitalar, ambulatorial ou nos serviços de emergência. Nesse sentido, é importante que além do atendimento curativo, os profissionais estejam aptos a realizarem a detecção precoce das complicações do câncer e do tratamento, bem como elaborarem estratégias para sua prevenção $(16,17)$.

Assim, o atendimento da enfermagem pode atuar de maneira significativa, tendo em vista, seu trabalho ser baseado na identificação de respostas humanas e no estabelecimento de estratégias que proporcionem a recuperação da saúde ou a melhoria do bemestar individual ou coletivo destes pacientes oncológicos, além disso, a equipe de enfermagem está próxima por mais tempo do paciente e seus familiares. $\mathrm{O}$ enfermeiro pode fazer uso do Processo de Enfermagem (PE), a fim de organizar ou sistematizar a assistência individualizada, holística com interação da equipe-cliente-família para os pacientes com câncer $(18,19)$.

De acordo com os resultados, observou-se uma pequena prevalência de pacientes oncológicos nesta unidade de emergência, com perfil de idade acima de 60 anos, na maioria do sexo masculino, em estágio inicial da doença oncológica, apresentando como queixa principal dor, náuseas e vômitos, recebendo tratamento medicamentoso sintomático por via endovenosa e a maioria teve alta do serviço de emergência após melhora dos sintomas.

O tratamento dos sintomas em pacientes com câncer é uma preocupação multiprofissional complexa e os serviços de emergência devem ter estrutura física e recursos humanos qualificados para atender os pacientes em emergências oncológicas. 


\section{REFERÊNCIAS}

1. Instituto Nacional de Câncer José Alencar Gomes da Silva (INCA). Coordenação de Prevenção e Vigilância Estimativa 2014: Incidência de Câncer no Brasil / Instituto Nacional de Câncer José Alencar Gomes da Silva, Coordenação de Prevenção e Vigilância. Rio de Janeiro: INCA; 2014. 124 p.

2. Ministério da Saúde. Instituto Nacional de Câncer. Estimativa 2012: Incidência de câncer no Brasil [Internet]. Rio de Janeiro: INCA; 2011 [citado 13 julho 2015]. Disponível em: http://www.inca.gov.br/ estimativa/2012/estimativa20122111.pdf

3. Gabriel J. Acute oncological emergencies. Nursing Standard [Internet]. 2012 [citado 12 julho 2015]; 27(4): 35-42 Disponível em: www. tiny.cc/csccn_oncology..

4. Sadik M, Ozlem K, Huseyin M, AliAyberk B, Ahmet S, Ozgur O. Attributes of cancer patients admitted to the emergency department in one year. World J Emerg Med. 2014; 5(2): 85-90.

5. Silva MM, Esteves LO, Moreira MC, Silva JA, Machado SC, Campos JF. Perfil de diagnósticos de enfermería en un hospital brasileño especializado en cuidados paliativos oncológicos. Cienc. enferm. 2013; XIX(1): 49-59.

6. Amaral AFS, Pulido KCS, Santos VLCG. Prevalência de lesões por friç̧ão em pacientes hospitalizados com câncer. Rev Esc Enferm USP. 2012; 46(Esp): 44-50.

7. Freire MEM, Sawada NO, França ISX, Costa SFG, Oliveira CDB. Qualidade de vida relacionada à saúde de pacientes com câncer avançado: uma revisão integrativa. Rev Esc Enferm USP. 2014; 48(2): 357-67.

8. Roncarati R, Camargo RMP, Rossetto EG, Matsuo T. Cuidados Paliativos num Hospital Universitário de Assistência Terciária: uma necessidade? Semina cienc. biol.
Saúde. 2003; 24: 37-48.

9. Cunha FF, Rêgo LP. Enfermagem diante da dor oncológica. Rev Dor. 2015; 16(2): 142-5.

10. Kadakia, et al. Antiemetic prescribing practices using a computerized physician order entry system. Support Care Cancer. 2014; 22(1): 217-223.

11. Fripp JC, Facchini LA, Silva SM. Characterizing a home and palliative care program in the Municipality of Pelotas, State of Rio Grande do Sul, Brazil: a contribution to full attention to cancer patients at the National Unified Health System. Epidemiol. Serv. Saúde. 2012; 21(1): 69-78.

12. Gaertner J, Frechen S, Sladek M, Ostgathe C, Voltz R. Palliative Care Consultation Service and Palliative Care Unit: Why Do We Need Both? The Oncologist. 2012; 17: 428-435.

13. Tow S. Tan, Aminah Jatoi End-of-Life Hospital Costs in Cancer Patients: Do Advance Directives or Routes of Hospital Admission Make a Difference? Oncology 2011; 80: 118-122.

14. Chan A, Soh D, Ko Y, Huang Y, Chiang J. Characteristics of unplanned hospital admissions due to drug-related problems in cancer patients. Support Care Cancer. 2014; 22: 1875-1881.

15. Seow H, Brazil K, Sussman J, Pereira J, Marshall D, Austin PC et al. Impact of community based, specialist palliative care teams on hospitalisations and emergency department visits late in life and hospital deaths: a pooled analysis. BMJ. 2014; 348: g3496.

16. Brooks GA, Abrams TA, Meyerhardt JA, Enzinger PC, Sommer K, Dalby CK, et al. Identification of Potentially Avoidable Hospitalizations in Patients with GI Cancer. Clin Oncol. 2014; 32: 496-503.

17. Silva MM, Moreira MC, Leite JL, Erdmann AL. Análise do cuidado de enfermagem e da participação dos familiares na atenção paliativa oncológica. Texto 
contexto-enferm. 2012; 21(3): 658-66.

18. Souza BF, Pires FH, Dewulf NLS, Inocenti A, Silva AEBC, Miasso A. Pacientes em uso de quimioterápicos: depressão e adesão ao tratamento. Rev Esc Enferm USP. 2013; 47(1): 61-8.
19. Nascimento LKAS, Medeiros ATN, Saldanha EA, Tourinho FSV, Santos VEP, Lira ALBC. Sistematização da assistência de enfermagem a pacientes oncológicos: uma revisão integrativa da literatura. Rev Gaucha Enferm. 2012; 33(1): 177-85. 